\title{
Action Principles for Relativistic Extended Magnetohydrodynamics: A Unified Theory of Magnetofluid Models
}

\author{
Yohei Kawazura, 1,2, a) George Miloshevich,3, b) and Philip J. Morrison ${ }^{3, c)}$ \\ ${ }^{1)}$ Graduate School of Frontier Sciences, The University of Tokyo, Kashiwa, Chiba 277-8561, \\ Japan \\ ${ }^{2)}$ Rudolf Peierls Centre for Theoretical Physics, University of Oxford, Oxford OX1 3NP, \\ $U K$ \\ ${ }^{3)}$ Department of Physics and Institute for Fusion Studies, The University of Texas at Austin, Austin, TX 78712, \\ USA
}

(Dated: 30 September 2018)

Two types of Eulerian action principles for relativistic extended magnetohydrodynamics (MHD) are formulated. With the first, the action is extremized under the constraints of density, entropy, and Lagrangian label conservation, which leads to a Clebsch representation for a generalized momentum and a generalized vector potential. The second action arises upon transformation to physical field variables, giving rise to a covariant bracket action principle, i.e., a variational principle in which constrained variations are generated by a degenerate Poisson bracket. Upon taking appropriate limits, the action principles lead to relativistic Hall MHD and well-known relativistic ideal MHD. For the first time, the Hamiltonian formulation of relativistic Hall MHD with electron thermal inertia (akin to [Comisso et al., Phys. Rev. Lett. 113, 045001 (2014)] for the electron-positron plasma) is introduced. This thermal inertia effect allows for violation of the frozen-in magnetic flux condition in marked contrast to nonrelativistic Hall MHD that does satisfy the frozenin condition. We also find violation of the frozen-in condition is accompanied by freezing-in of an alternative flux determined by a generalized vector potential. Finally, we derive a more general $3+1$ Poisson bracket for nonrelativistic extended MHD, one that does not assume smallness of the electron ion mass ratio.

PACS numbers: 95.30.Qd, 95.30.Sf, 52.27.Ny, 52.30.Cv, 03.30.+p, 03.50.-z

\section{INTRODUCTION}

The early discovery of action principles (AP)s and associated Hamiltonian structure, undoubtably of groundbreaking importance in the history of physics, has unified existing physical models and provided a means for the development of new models. In physics it is now believed that an empirically derived physical model, devoid of phenomenological constitutive relations, would not be justified unless an underlying AP exists. In addition to mathematical elegance, APs are of practical importance for seeking invariants via symmetries using Noether's theorem ${ }^{1}$ (see, e.g., Refs. 2 and 3 for plasma examples), obtaining consistent approximations (e.g., Ref. 4), and developing numerical algorithms (e.g., Refs. 5-7).

In this paper, we obtain APs for relativistic magnetofluid models. The key ingredient for constructing APs for a fluidlike systems is a means for implementing constraints, because direct extremization yields trivial equations of motion. There are various formalisms available, depending on how the constraints are implemented. One is to follow Lagrange ${ }^{8}$ and incorporate constraints into the definition of the variables. This procedure is invoked when using Lagrangian coordinates with the time evolution of variables (fluid element attributes) (e.g., density and entropy) described a priori by conservation of differential forms along stream lines. APs in the Lagrangian coordinates have been obtained for the nonrelativistic neutral

\footnotetext{
${ }^{a)}$ Electronic mail: yohei.kawazura@physics.ox.ac.uk

b)Electronic mail: gmilosh@physics.utexas.edu

c)Electronic mail: morrison@physics.utexas.edu
}

fluid, magnetohydrodynamics (MHD), ${ }^{9}$ and various generalized magnetofluid models (e.g. extended MHD (XMHD), inertial MHD (IMHD), and Hall MHD (HMHD)), ${ }^{4,10,11}$ as well as for the relativistic neutral fluid ${ }^{12-14}$ and MHD. ${ }^{15,16}$ In obtaining such formulations several complications arise, e.g., the inference of the appropriate Lagrangian variables, the map between the Lagrangian and Eulerian coordinates in the relativistic case, ${ }^{16}$ and the existence of multiple flow characteristics for generalized magnetofluid models. ${ }^{4,11}$

A second type of AP, one that is formulated in terms Eulerian variables, implements the constraints via Lagrange multipliers, and in this way extremization of the action can lead to correct equations of motion. ${ }^{17,18}$ Upon enforcing the constraints of conservation of density, entropy, and a Lagrangian label, ${ }^{18}$ this procedure was recently used to obtain nonrelativistic HMHD. ${ }^{19}$ Then, this formulation for HMHD was used to regularize the singular limit to MHD by a renormalization of variables, thereby obtaining an AP for MHD. ${ }^{19}$ For the relativistic neutral fluid, the velocity norm (lightcone) condition ( $u^{\mu} u_{\mu}=1$ with fluid four-velocity $u^{\mu}$ ) is required as another constraint. ${ }^{20-24}$ Instead of taking the limit from HMHD with renormalization, there are alternative formulations for nonrelativistic $^{25}$ and relativistic ${ }^{26,27} \mathrm{MHD}$, in which the Ohm's law or the induction equation per se is employed as a constraint.

A third type of AP, one of general utility that incorporates a covariant Poisson bracket in terms of Eulerian variables, was introduced in Ref. 28. Instead of including the constraints in the action with Lagrange multipliers, the constraints are implemented via the degeneracy of a Poisson bracket that effects constrained variations. In addition to the neutral fluid, such Poisson bracket APs have been described for particle me- 
chanics, electromagnetism, the Vlasov-Maxwell system, and the gravitational field. ${ }^{28}$ Most recently, this kind of action was obtained for relativistic MHD. ${ }^{29}$

From Table I, which summarizes the aforementioned APs, we see there are missing pieces: the APs for fluid-dynamical systems are (i) the Lagrangian AP, (ii) Eulerian constrained least AP, and (iii) the Eulerian bracket AP, for relativistic generalized magnetofluid models. In this paper, we formulate the latter two APs: (ii) and (iii), and show that they are related by variable transformation. Then we derive APs for HMHD and MHD by taking limits of the XMHD AP. Relativistic HMHD is derived for the first time in the present study by this method. Also, we show that the nonrelativistic limit of the bracket AP gives nonrelativistic XMHD as a Hamiltonian system.

This paper is organized as follows. In Sec. II we formulate a constrained least AP for relativistic XMHD. In Sec. III the bracket AP is derived by a transformation of phase space variables in the constrained least AP. In Sec. IV we derive relativistic HMHD and MHD by taking limits of the bracket AP for XMHD. These results are used in Sec. V where remarkable features of relativistic HMHD pertaining to collisionless reconnection are considered. In Sec. VI, the nonrelativistic limit of the bracket AP is shown. Finally in Sec. VII we conclude.

\section{CONSTRAINED LEAST ACTION PRINCIPLE}

Consider a relativistic plasma consisting of positively and negatively charged particles with masses $m_{+}$and $m_{-}$, where subscript signs denote species labels, and assume the Minkowski spacetime with the metric tensor $\operatorname{diag}(1,-1,-1,-1)$. In addition, a proper charge neutrality condition is imposed so that rest frame particle number densities of each species satisfy $n_{+}=n_{-}=n .^{30}$ The four-velocities of each species are denoted by ${u_{ \pm}}^{\mu}$, which obey the velocity norm conditions

$$
u_{ \pm}{ }^{\mu} u_{ \pm \mu}=1 .
$$

Using the four-velocities ${u_{ \pm}}^{\mu}$, the four-center of mass velocity and the four-current density can be written as

$$
\begin{aligned}
u^{\mu} & =\left(m_{+} / m\right) u_{+}{ }^{\mu}+\left(m_{-} / m\right) u_{-}{ }^{\mu}, \\
J^{\mu} & =e\left(u_{+}{ }^{\mu}-u_{-}{ }^{\mu}\right),
\end{aligned}
$$

respectively, with $m=m_{+}+m_{-}$and the electric charge $e$. The time and space components of these fields are written as $u^{\mu}=(\gamma, \gamma \mathbf{v} / c)$ and $J^{\mu}=\left(\rho_{q}, \mathbf{J}\right)$ with speed of light $c$, Lorentz factor $\gamma=1 / \sqrt{1-(|\mathbf{v}| / c)^{2}}$, and charge density $\rho_{q}$. The thermodynamic variables needed are the energy density $\rho_{ \pm}$, the enthalpy density $h_{ \pm}$, the entropy density $\sigma_{ \pm}$, and the isotropic pressure $p_{ \pm}$. These are related by $n h_{ \pm}=p_{ \pm}+\rho_{ \pm}=$ $n\left(\partial \rho_{ \pm} / \partial n\right)+\sigma_{ \pm}\left(\partial \rho_{ \pm} / \partial \sigma_{ \pm}\right) .{ }^{28}$ We also define total energy density $\rho=\rho_{+}+\rho_{-}$and total pressure $p=p_{+}+p_{-}$.

Adding the continuity equations for each species together leads an equation for $n$,

$$
\partial_{v}\left(n u^{v}\right)=0,
$$

while the adiabatic equations of each species can be written as

$$
\partial_{v}\left(\sigma_{ \pm} u_{ \pm}{ }^{\nu}\right)=0 .
$$

In addition to the above constraint equations we include conservations of the Lagrangian labels $\varphi_{ \pm}$,

$$
u_{ \pm v} \partial^{v} \varphi_{ \pm}=0 \text {. }
$$

The full set of independent variables of our action are chosen to be $\left(u^{\mu}, J^{\mu}, n, \sigma_{ \pm}, \varphi_{ \pm}, A^{\mu}\right)$, where $A^{\mu}$ is a four-vector potential that defines a Faraday tensor $\mathcal{F}^{\mu \nu}=\partial^{\mu} A^{\nu}-\partial^{v} A^{\mu}$. Here we consider CGS unit getting rid of a factor $1 / 4 \pi$ in the Faraday tensor by renormalization (i.e., $\mathcal{F}^{\mu \nu} / 4 \pi \rightarrow \mathcal{F}^{\mu \nu}$ ). In a manner similar to that of Lin's formalism ${ }^{18}$ for the nonrelativistic neutral fluid, we bring (4), (5), and (6) into an action as constraints as follows:

$$
\begin{aligned}
& S\left[u, J, n, \sigma_{ \pm}, A, \varphi_{ \pm}\right]= \\
& \int\left\{\sum_{ \pm}\left[-\frac{1}{2} n h_{ \pm} u_{ \pm v} u_{ \pm}{ }^{v}+\frac{1}{2}\left(p_{ \pm}-\rho_{ \pm}\right)\right]-J^{v} A_{v}\right. \\
& \quad-\frac{1}{4}\left(\partial^{\mu} A^{v}-\partial^{v} A^{\mu}\right)\left(\partial_{\mu} A_{v}-\partial_{v} A_{\mu}\right)-\phi \partial^{v}\left(n u_{v}\right) \\
& \left.\quad-\sum_{ \pm}\left[\eta_{ \pm} \partial^{v}\left(\sigma_{ \pm} u_{ \pm v}\right)-\lambda_{ \pm} u_{ \pm v} \partial^{v} \varphi_{ \pm}\right]\right\} \mathrm{d}^{4} x,
\end{aligned}
$$

where $\sum_{ \pm}$is summation over species, and $\phi, \eta_{ \pm}$, and $\lambda_{ \pm}$are Lagrange multipliers. The first and second terms of (7) are the fluid part for each species, the third term is an interaction between the fluid and the electromagnetic (EM) field, the fourth term is the pure EM part, and the other terms represent the constraints. The velocity norm conditions (1) will be imposed after variation of the action. ${ }^{31}$

Variation of the action, i.e., setting $\delta S=0$, gives

$$
\begin{aligned}
& \delta u_{v}: n h u^{v}+\frac{\Delta h}{e} J^{v}=n \partial^{v} \phi+\sum_{ \pm}\left(\sigma_{ \pm} \partial^{v} \eta_{ \pm}+\lambda_{ \pm} \partial^{v} \varphi_{ \pm}\right) \\
& \delta J_{v}: A^{v}+\frac{\Delta h}{e} u^{v}+\frac{h^{\dagger}}{n e^{2}} J^{v}= \\
& \quad \sum_{ \pm}\left[ \pm \frac{m_{\mp}}{m e n}\left(\sigma_{ \pm} \partial^{v} \eta_{ \pm}+\lambda_{ \pm} \partial^{v} \varphi_{ \pm}\right)\right] \\
& \delta \sigma_{ \pm}: u_{ \pm v} \partial^{v} \eta_{ \pm}=\frac{\partial \rho_{ \pm}}{\partial \sigma_{ \pm}} \\
& \delta \varphi_{ \pm}: \partial^{v}\left(\lambda_{ \pm} u_{ \pm v}\right)=0 \\
& \delta A^{v}: J_{v}=\partial^{\mu} \mathcal{F}_{\mu v} \\
& \delta n: n u_{v} \partial^{v} \phi=A^{v} J_{v}+n \sum_{ \pm} \frac{\partial \rho_{ \pm}}{\partial n},
\end{aligned}
$$

with $h:=h_{+}+h_{-}, \Delta h:=\left(m_{-} / m\right) h_{+}-\left(m_{+} / m\right) h_{-}$, and $h^{\dagger}=$ $\left(m_{-}^{2} / m^{2}\right) h_{+}+\left(m_{+}^{2} / m^{2}\right) h_{-}$. Using (4), (5), (6), and (8)-(13), the momentum equation and generalized Ohm's law are obtained:

$$
\begin{aligned}
& \partial_{v}\left[n h u^{\mu} u^{v}+\frac{\Delta h}{e}\left(u^{\mu} J^{v}+J^{\mu} u^{v}\right)+\frac{h^{\dagger}}{n e^{2}} J^{\mu} J^{v}\right] \\
& =\partial^{\mu} p+J^{v} \mathcal{F}^{\mu}{ }_{\nu}, \\
& \partial_{v}\left[n(\Delta h) u^{\mu} u^{v}+\frac{h^{\dagger}}{e}\left(u^{\mu} J^{v}+J^{\mu} u^{v}\right)+\frac{\Delta h^{\sharp}}{n e^{2}} J^{\mu} J^{v}\right] \\
& =\frac{m_{-}}{m} \partial^{\mu} p_{+}-\frac{m_{+}}{m} \partial^{\mu} p_{-}+e n u^{v} \mathcal{F}^{\mu}{ }_{v}-\frac{m_{+}-m_{-}}{m} J^{v} \mathcal{F}^{\mu}{ }_{\nu},
\end{aligned}
$$


TABLE I. Summary of APs for fluid-dynamical systems. The bold faces indicate APs which have not formulated until the present study.

\begin{tabular}{|c|c|c|c|}
\hline & Constrained least AP & Covariant bracket AP & Lagrangian description AP \\
\hline $\begin{array}{c}\text { Nonrelativistic } \\
\text { fluid }\end{array}$ & Lin (1963) & Present study & Lagrange (1788) \\
\hline $\begin{array}{c}\text { Nonrelativistic } \\
\text { MHD }\end{array}$ & $\begin{array}{c}\text { Yoshida \& Hameiri (2013) (renormalization \& limit from HMHD) } \\
\text { Webb et al. (2014) (Ohm's law constraint) }\end{array}$ & Present study & Newcomb (1962) \\
\hline $\begin{array}{c}\text { Nonrelativistic } \\
\text { XMHD }\end{array}$ & Yoshida \& Hameiri (2013) (HMHD) & Present study & $\begin{array}{c}\text { Keramidas Charidakos } \\
\text { et al. (2014) }\end{array}$ \\
\hline $\begin{array}{c}\text { Relativistic } \\
\text { fluid }\end{array}$ & Schutz (1970) & Marsden et al. (1986) & $\begin{array}{c}\text { Dewar (1977) } \\
\text { Salmon (1988) }\end{array}$ \\
\hline $\begin{array}{c}\text { Relativistic } \\
\text { MHD }\end{array}$ & $\begin{array}{c}\text { Present study (renormalization \& limit from HMHD) } \\
\text { Bekenstein \& Oron (2000) (Ohm's law constraint) }\end{array}$ & D'Avignon et al. (2015) & $\begin{array}{c}\text { Achterberg (1983) } \\
\text { Kawazura et al. (2014) }\end{array}$ \\
\hline $\begin{array}{c}\text { Relativistic } \\
\text { XMHD }\end{array}$ & Present study & Present study & unknown \\
\hline
\end{tabular}

with $\Delta h^{\sharp}=\left(m_{-}^{3} / m^{3}\right) h_{+}-\left(m_{+}^{3} / m^{3}\right) h_{-}$. These are equivalent to the relativistic XMHD equations previously formulated by Koide. ${ }^{30,32}$ The generalized Ohm's law of (14) can be rewritten as

$$
e u_{v} \mathcal{F}^{\star \mu \nu}-\frac{J_{v}}{n} F^{\dagger \mu v}=\frac{m_{-}}{m}\left(T_{+} \partial^{\mu} \frac{\sigma_{+}}{n}\right)-\frac{m_{+}}{m}\left(T_{-} \partial^{\mu} \frac{\sigma_{-}}{n}\right),
$$

with

$$
\begin{aligned}
A^{\dagger^{v}} & =\frac{m_{+}-m_{-}}{m} A^{v}-\frac{h^{\dagger}}{e} u^{v}-\frac{\Delta h^{\sharp}}{n e^{2}} J^{v}, \\
\mathcal{F}^{\star \mu \nu} & =\partial^{\mu} A^{\star \nu}-\partial^{v} A^{\star \mu} \text { and } \mathcal{F}^{\dagger \mu \nu}=\partial^{\mu} A^{\dagger^{\nu}}-\partial^{v} A^{\dagger \mu},
\end{aligned}
$$

where a generalized vector potential $A^{\star}$ is defined by

$$
A^{\star v}=A^{v}+\frac{\Delta h}{e} u^{v}+\frac{h^{\dagger}}{n e^{2}} J^{v} .
$$

Note, the following must hold as an identity,

$$
\partial^{\mu}\left(\epsilon_{\mu \nu \rho \sigma} \mathcal{F}^{\star \rho \sigma}\right)=0
$$

where $\epsilon_{\mu \nu \rho \sigma}$ is the four-dimensional Levi-Civita symbol. Upon taking the four-dimensional curl of (16), we obtain the generalized induction equation

$$
\begin{aligned}
& e\left[\partial^{\mu}\left(u_{\lambda} \mathcal{F}^{\star \nu \lambda}\right)-\partial^{v}\left(u_{\lambda} \mathcal{F}^{\star \mu \lambda}\right)\right]-\left[\partial^{\mu}\left(\frac{J_{\lambda}}{n} \mathcal{F}^{\dagger^{\nu \lambda}}\right)\right. \\
& \left.-\partial^{v}\left(\frac{J_{\lambda}}{n} \mathcal{F}^{\dagger \mu \lambda}\right)\right]-\frac{m_{-}}{m}\left[\partial^{\mu} T_{+} \partial^{v}\left(\frac{\sigma_{+}}{n}\right)-\partial^{v} T_{+} \partial^{\mu}\left(\frac{\sigma_{+}}{n}\right)\right] \\
& +\frac{m_{+}}{m}\left[\partial^{\mu} T_{-} \partial^{v}\left(\frac{\sigma_{-}}{n}\right)-\partial^{v} T_{-} \partial^{\mu}\left(\frac{\sigma_{-}}{n}\right)\right]=0 .
\end{aligned}
$$

Next, upon combining (14) and (15) we obtain equations for the canonical momenta ${ }^{33}$ of each species

$$
u_{ \pm v}\left(\partial^{\mu} \wp_{ \pm}^{v}-\partial^{v} \wp_{ \pm}^{\mu}\right)+T_{ \pm} \partial^{\mu}\left(\frac{\sigma_{ \pm}}{n}\right)=0
$$

where $\wp_{ \pm}{ }^{v}=h_{ \pm} u_{ \pm}{ }^{v} \pm e A^{v}$. Several simplifications have been proposed to make these equations tractable; ${ }^{30,32}$ e.g., the assumption of $\Delta h=0$ (i.e., $h_{+}=\left(m_{+} / m\right) h$ and $\left.h_{-}=\left(m_{-} / m\right) h\right)$ and/or the usage of the velocity norm condition $u_{\mu} u^{\mu}=1$ with
(2) instead of (1). The latter condition requires $J_{\mu} J^{\mu}=0$ to be consistent with (1) (referred to as the "break down condition" in Ref. 30). Such a simplified model has recently come into usage. ${ }^{34-36}$ Imposing $\Delta h=0$ on the action (7) and/or replacing (1) by $u_{\mu} u^{\mu}=1$ and $J_{\mu} J^{\mu}=0$, this simplified model is directly obtained from the AP.

\section{COVARIANT BRACKET ACTION PRINCIPLE}

Now we construct our covariant action principle. To this end we define a kinetic momentum $\mathrm{m}^{v}=n h u^{v}$ and a generalized momentum $\mathrm{m}^{\star v}=\mathrm{m}^{v}+(\Delta h / e) J^{v}$. Then (8) and (9) can then be viewed as the Clebsch representations for $\mathrm{m}^{\star \nu}$ and $A^{\star v}$. The reason for introducing these new field variables is that the action (7) takes a beautiful form in terms of them:

$$
\begin{aligned}
S=\int\left[\frac{\mathfrak{m}^{\star v} \mathrm{~m}_{v}}{2 n h}+\sum_{ \pm} \frac{1}{2}\left(p_{ \pm}-\rho_{ \pm}\right)\right. \\
\left.\quad-\frac{1}{4}\left(\partial^{\mu} A^{\star v}-\partial^{v} A^{\star \mu}\right)\left(\partial_{\mu} A_{v}-\partial_{v} A_{\mu}\right)\right] \mathrm{d}^{4} x .
\end{aligned}
$$

Interestingly, upon letting $\mathrm{m}^{\star v} \rightarrow \mathrm{m}^{v}$ and $A^{\star v} \rightarrow A^{v}$, the action (20) becomes identical to the recently proposed relativistic MHD action of Ref. 29. When the simplification $\Delta h \rightarrow 0$ is imposed, $m^{\star^{v}}$ becomes the kinetic momentum and $A^{\star^{v}}$ is decoupled from the kinetic momentum (the nonrelativistic version of such a vector potential was previously proposed for nonrelativistic IMHD ${ }^{10}$ and $\mathrm{XMHD}^{37,38}$ ). In other words, the difference of the thermal inertiae between species (i.e. $\Delta h$ ) intertwines the kinetic momentum field and the EM field. Since the nonrelativistic limit $\left(h_{+} \rightarrow m_{+} c^{2}\right.$ and $\left.h_{-} \rightarrow m_{-} c^{2}\right)$ results in $\Delta h \rightarrow 0$, such a coupling is distinctive of the relativistic two-fluid plasma.

For our covariant action it is convenient to use the Clebsch variables

$$
z=\left(n, \phi, \sigma_{ \pm}, \eta_{ \pm}, \lambda_{ \pm}, \varphi_{ \pm}\right)
$$

as the independent variables of the action (20). With these variables all of the dynamical equations (4), (5), (6), (10), 
(11), and (13) are derived from the least AP (i.e. $\delta S=0$ ). In terms of $z$ we can simply restate the AP of Sec. II as a canonical covariant bracket version of the formalism of Refs. 28 and 29. A canonical Poisson bracket is defined for functionals $F$ and $G$ as

$$
\begin{aligned}
&\{F, G\}_{\text {canonical }}= \int \frac{\delta F}{\delta z} \mathcal{J}_{\mathrm{c}} \frac{\delta G}{\delta z} \mathrm{~d}^{4} x \\
&=\int\left[\frac{\delta F}{\delta \phi} \frac{\delta G}{\delta n}-\frac{\delta G}{\delta \phi} \frac{\delta F}{\delta n}\right. \\
&+\sum_{ \pm}\left(\frac{\delta F}{\delta \eta_{ \pm}} \frac{\delta G}{\delta \sigma_{ \pm}}-\frac{\delta G}{\delta \eta_{ \pm}} \frac{\delta F}{\delta \sigma_{ \pm}}\right. \\
&\left.\left.+\frac{\delta F}{\delta \varphi_{ \pm}} \frac{\delta G}{\delta \lambda_{ \pm}}-\frac{\delta G}{\delta \varphi_{ \pm}} \frac{\delta F}{\delta \lambda_{ \pm}}\right)\right] \mathrm{d}^{4} x
\end{aligned}
$$

where $\mathcal{J}_{\mathrm{c}}$ is the symplectic matrix and $\delta F / \delta z$ denotes the functional derivative obtained by linearizing a functional, e.g.

$$
\delta F=\int \delta n \frac{\delta F}{\delta n} \mathrm{~d}^{4} x
$$

(See Ref. 39 for review.) Since $\mathcal{J}_{\mathrm{c}}$ is non-degenerate, with $(n, \phi),\left(\sigma_{ \pm}, \eta_{ \pm}\right)$, and $\left(\lambda_{ \pm}, \varphi_{ \pm}\right)$being canonically conjugate pairs, the least AP is equivalent to a bracket AP, i.e., $\{F[z], S\}_{\text {canonical }}=0$ where $F[z]$ is an arbitrary functional of $z$, is equivalent to $\delta S=0$.

Transformation to new "physical" independent variables defined by

$$
\bar{z}=\left(n, \sigma_{ \pm}, \mathfrak{m}^{\star v}, \mathcal{F}^{\star \mu v}\right)
$$

yields a noncanonical covariant bracket because the $\bar{z}$ are not canonical variables. To transform the bracket of (21) we consider functionals that satisfy $\bar{F}[\bar{z}]=F[z]$, and calculate functional derivatives by the chain rule

$$
\begin{aligned}
\frac{\delta F}{\delta n}= & \frac{\delta \bar{F}}{\delta n}+\frac{\delta \bar{F}}{\delta m^{\star \nu}} \partial^{v} \phi \\
& +\frac{2 m_{-} \sigma_{+}}{m e n^{2}} \partial^{\mu}\left(\frac{\delta \bar{F}}{\delta \mathcal{F}^{\star \mu \nu}} \partial^{v} \eta_{+}\right)+\frac{2 m_{-} \lambda_{+}}{m e n^{2}} \partial^{\mu}\left(\frac{\delta \bar{F}}{\delta \mathcal{F}^{\star \mu \nu}} \partial^{v} \varphi_{+}\right) \\
& -\frac{2 m_{+} \sigma_{-}}{m e n^{2}} \partial^{\mu}\left(\frac{\delta \bar{F}}{\delta \mathcal{F}^{\star \mu \nu}} \partial^{v} \eta_{-}\right)-\frac{2 m_{+} \lambda_{-}}{m e n^{2}} \partial^{\mu}\left(\frac{\delta \bar{F}}{\delta \mathcal{F}^{\star \mu \nu}} \partial^{v} \varphi_{-}\right), \\
\frac{\delta F}{\delta \phi}= & -\partial^{v}\left(n \frac{\delta \bar{F}}{\delta m^{\star \nu}}\right), \\
\frac{\delta F}{\delta \sigma_{ \pm}}= & \frac{\delta \bar{F}}{\delta \sigma_{ \pm}}+\frac{\delta \bar{F}}{\delta m^{\star \nu}} \partial^{v} \eta_{ \pm} \mp \frac{2 m_{\mp}}{m e n} \partial^{\mu}\left(\frac{\delta \bar{F}}{\delta \mathcal{F}^{\star \mu \nu}} \partial^{v} \eta_{ \pm}\right) \\
\frac{\delta F}{\delta \eta_{ \pm}}= & -\partial^{v}\left(\sigma_{ \pm} \frac{\delta \bar{F}}{\delta m^{\star \nu}}\right) \pm \frac{2 m_{\mp}}{m e} \partial^{\mu}\left(\frac{\delta \bar{F}}{\delta \mathcal{F}^{\star \mu \nu}} \partial^{\nu} \frac{\sigma_{ \pm}}{n}\right) \\
\frac{\delta F}{\delta \lambda_{ \pm}}= & \frac{\delta \bar{F}}{\delta m^{\star \nu}} \partial^{v} \varphi_{ \pm} \mp \frac{2 m_{\mp}}{m e n} \partial^{\mu}\left(\frac{\delta \bar{F}}{\delta \mathcal{F}^{\star \mu \nu}} \partial^{v} \varphi_{ \pm}\right) \\
\frac{\delta F}{\delta \varphi_{ \pm}}= & -\partial^{v}\left(\lambda_{ \pm} \frac{\delta \bar{F}}{\delta m^{\star \nu}}\right) \pm \frac{2 m_{\mp}}{m e} \partial^{\mu}\left(\frac{\delta \bar{F}}{\delta \mathcal{F} \star \mu \nu} \partial^{v} \frac{\lambda_{ \pm}}{n}\right)
\end{aligned}
$$

Substituting these into (21) gives the following noncanonical Poisson bracket,

$$
\begin{aligned}
\{\bar{F}, \bar{G}\}_{\mathrm{XMHD}} & =-\int\left\{n\left(\frac{\delta \bar{G}}{\delta m^{\star \nu}} \partial^{\nu} \frac{\delta \bar{F}}{\delta n}-\frac{\delta \bar{F}}{\delta m^{\star \nu}} \partial^{\nu} \frac{\delta \bar{G}}{\delta n}\right)\right. \\
& +m^{\star \nu}\left(\frac{\delta \bar{G}}{\delta m^{\star \mu}} \partial^{\mu} \frac{\delta \bar{F}}{\delta m^{\star \nu}}-\frac{\delta \bar{F}}{\delta m^{\star \mu}} \partial^{\mu} \frac{\delta \bar{G}}{\delta m^{\star \nu}}\right) \\
& +\sum_{ \pm}\left[\sigma_{ \pm}\left(\frac{\delta \bar{G}}{\delta m^{\star \nu}} \partial^{\nu} \frac{\delta \bar{F}}{\delta \sigma_{ \pm}}-\frac{\delta \bar{F}}{\delta m^{\star \nu}} \partial^{\nu} \frac{\delta \bar{G}}{\delta \sigma_{ \pm}}\right)\right. \\
& \left. \pm \frac{2 m_{\mp}}{m e}\left(\frac{\delta \bar{F}}{\delta \sigma_{ \pm}} \partial^{\mu} \frac{\delta \bar{G}}{\delta \mathcal{F}^{\star \mu \nu}}-\frac{\delta \bar{G}}{\delta \sigma_{ \pm}} \partial^{\mu} \frac{\delta \bar{F}}{\delta \mathcal{F}^{\star \mu \nu}}\right) \partial^{\nu} \frac{\sigma_{ \pm}}{n}\right] \\
& +2\left(\frac{\delta \bar{F}}{\delta m^{\star \lambda}} \partial^{\mu} \frac{\delta \bar{G}}{\delta \mathcal{F} \star^{\mu \nu}}-\frac{\delta \bar{G}}{\delta m^{\star \lambda}} \partial^{\mu} \frac{\delta \bar{F}}{\delta \mathcal{F}^{\star \mu \nu}}\right) \mathcal{F}^{\star \nu \lambda} \\
& \left.+\frac{4}{n e}\left(\partial^{\mu} \frac{\delta \bar{F}}{\delta \mathcal{F}^{\star \mu \nu}}\right)\left(\partial^{\lambda} \frac{\delta \bar{G}}{\delta \mathcal{F}^{\star \lambda \kappa}}\right) \mathcal{F}^{\dagger \kappa \nu}\right\} \mathrm{d}^{4} x .
\end{aligned}
$$

The fluid parts (the first three terms) of (23) correspond to the covariant Poisson bracket for the neutral fluid given in Ref. 28. Next, in order to use this bracket in a variational sense, the action (20) is considered to be the functional of $\left(n, \sigma_{ \pm}, m^{\star v}, \mathcal{F}^{\star \mu \nu}\right)$, i.e. $\bar{S}[\bar{z}]$, and its functional derivatives are calculated as

$$
\begin{gathered}
\frac{\delta \bar{S}}{\delta m^{\star \nu}}=u_{v}, \quad \frac{\delta \bar{S}}{\delta \mathcal{F}^{\star \mu \nu}}=-\frac{1}{2} \mathcal{F}_{\mu v}, \quad \frac{\delta \bar{S}}{\delta \sigma_{ \pm}}=-\frac{\partial \rho_{ \pm}}{\partial \sigma_{ \pm}} \\
\frac{\delta \bar{S}}{\delta n}=h_{+} \frac{m_{-}}{m e n} J_{v}\left(u^{v}+\frac{m_{-}}{m e n} J^{v}\right)-h_{-} \frac{m_{+}}{m e n} J_{v}\left(u^{v}-\frac{m_{+}}{m e n} J^{v}\right) \\
-\frac{\partial \rho_{+}}{\partial n}-\frac{\partial \rho_{-}}{\partial n}+\frac{\Delta h}{n e} J_{v} u^{v}+\frac{h^{\dagger}}{n^{2} e^{2}} J_{v} J^{v} .
\end{gathered}
$$

Then equations (4), (5), (14), and (19) follow from $\{\bar{F}[\bar{z}], \bar{S}\}=$ 0 for all $\bar{F}$.

Here we must remark that the equations obtained from the bracket action principle are not closed unless (18) is imposed. Although (18) is automatically satisfied by the Clebsch variable definition of $\mathcal{F}^{\star \mu \nu}$, it does not emerge from the bracket AP. Therefore, the bracket AP, only by itself, does not give the closed set of equations. This is a marked difference between a Hamiltonian formalism of nonrelativistic MHD; ${ }^{40,41}$ although $\nabla \cdot \mathbf{B}=0$ is not derived from the Hamiltonian equation, the obtained equations are closed even if $\nabla \cdot \mathbf{B} \neq 0$. On the other hand, in the relativistic case, if (18) is abandoned, we lose $\partial_{t} \mathbf{B}=-c \nabla \times \mathbf{E}$ as well.

There may be two remedies for this problem. One is to define a Faraday tensor that builds-in the Ohm's law (16) and consider (18) as a dynamical equation of the new Faraday tensor $^{29,42}$. This strategy, however, is difficult because the Ohm's law (16) is more complicated than that of relativistic MHD, and then it is hard to formulate the appropriate Faraday tensor. A second approach is to transform $\mathcal{F}^{\star \mu \nu}$ to $A^{\star \mu}$ so as to make the bracket action principle yield Ohm's law instead of the induction equation. To write the bracket of (23) in terms of $A^{\star \mu}$ we consider the functional chain rule to relate functional derivatives with respect to $\mathcal{F}^{\star \mu \nu}$ with those with respect 
to $A^{\star \mu}$, i.e.

$$
2 \partial_{v} \frac{\delta \bar{G}}{\delta \mathcal{F}^{\star \mu \nu}}=\frac{\delta \bar{G}}{\delta A^{\star \mu}} .
$$

Using (24), one can eliminate $\mathcal{F}^{\star \mu \nu}$ from the Poisson bracket (23) while introducing the variable $A^{\star \mu}$. This will give a bracket where the Ohm's law (16) is obtained directly. The transformation (24) yields

$$
\begin{aligned}
\{\bar{F}, \bar{G}\}_{\mathrm{XMHD}}= & -\int\left\{n\left(\frac{\delta \bar{G}}{\delta \mathfrak{m}^{\star \nu}} \partial^{\nu} \frac{\delta \bar{F}}{\delta n}-\frac{\delta \bar{F}}{\delta \mathfrak{m}^{\star \nu}} \partial^{\nu} \frac{\delta \bar{G}}{\delta n}\right)\right. \\
+ & \mathrm{m}^{\star \nu}\left(\frac{\delta \bar{G}}{\delta \mathrm{m}^{\star \mu}} \partial^{\mu} \frac{\delta \bar{F}}{\delta \mathrm{m}^{\star \nu}}-\frac{\delta \bar{F}}{\delta \mathrm{m}^{\star \mu}} \partial^{\mu} \frac{\delta \bar{G}}{\delta \mathrm{m}^{\star \nu}}\right) \\
& +\sum_{ \pm}\left[\sigma_{ \pm}\left(\frac{\delta \bar{G}}{\delta \mathfrak{m}^{\star \nu}} \partial^{\nu} \frac{\delta \bar{F}}{\delta \sigma_{ \pm}}-\frac{\delta \bar{F}}{\delta \mathfrak{m}^{\star \nu}} \partial^{\nu} \frac{\delta \bar{G}}{\delta \sigma_{ \pm}}\right)\right. \\
& \left.\mp \frac{m_{\mp}}{m e}\left(\frac{\delta \bar{F}}{\delta \sigma_{ \pm}} \frac{\delta \bar{G}}{\delta A^{\star \nu}}-\frac{\delta \bar{G}}{\delta \sigma_{ \pm}} \frac{\delta \bar{F}}{\delta A^{\star \nu}}\right) \partial^{\nu} \frac{\sigma_{ \pm}}{n}\right] \\
& +\left(\frac{\delta \bar{G}}{\delta m^{\star \nu}} \frac{\delta \bar{F}}{\delta A^{\star \mu}}-\frac{\delta \bar{F}}{\delta m^{\star \nu}} \frac{\delta \bar{G}}{\delta A^{\star \mu}}\right) \mathcal{F}^{\star \mu \nu} \\
& \left.-\frac{1}{n e} \frac{\delta \bar{F}}{\delta A^{\star \mu}} \frac{\delta \bar{G}}{\delta A^{\star \nu}} \mathcal{F}^{\dagger \mu \nu}\right\} \mathrm{d}^{4} x .
\end{aligned}
$$

Ohm's law follows from $\left\{A^{\star \alpha}, \bar{S}\right\}_{\mathrm{XMHD}}=0$ with $\delta \bar{S} / \delta A^{\star \mu}=$ $J_{\mu}$; the other equations are unaltered so the system is closed.

The noncanonical bracket of (25) has the form

$$
\{\bar{F}, \bar{G}\}_{\mathrm{XMHD}}=\int \frac{\delta \bar{F}}{\delta \bar{z}} \mathcal{J} \frac{\delta \bar{G}}{\delta \bar{z}} \mathrm{~d}^{4} x,
$$

with a new Poisson operator $\mathcal{J}$. However, because the transformation $z \mapsto \bar{z}$ is not invertible, the Poisson operator $\mathcal{J}$ is degenerate. Since the bracket AP, $\{\bar{F}[\bar{z}], \bar{S}\}=0$, is equivalent to $\mathcal{J} \delta \bar{S} / \delta \bar{z}=0$, because of this degeneracy it is no longer true that $\mathcal{J} \delta \bar{S} / \delta \bar{z}=0$ is identical to $\delta S=0$. In this way the constraints of the action (7) are transferred to the degeneracy of the Poisson bracket. ${ }^{28,29}$

Before closing this section, let us make a remark about the alternative expression of the EM field. The Faraday tensor may be decomposed as $\mathcal{F}^{\mu \nu}=\epsilon^{\mu \nu \lambda \sigma} b_{\lambda} u_{\sigma}+u^{\mu} e^{\nu}-u^{v} e^{\mu}$ with a magnetic field like four vector $b^{\nu}=u_{\mu} e^{\mu \nu \lambda \sigma} \mathcal{F}_{\lambda \sigma}$ and a electric field like four vector $e^{v}=u_{\mu} \mathcal{F}^{\mu \nu} .{ }^{42-44}$ This decomposition is especially useful in the relativistic MHD because the standard Ohm's law is equivalent to $e^{v}=0$, and thus EM field is concisely expressed only by $b^{\nu}$. In the context of the action principle, D'Avignon et al. formulated the bracket AP for the relativistic MHD using $b^{v}{ }^{29}$ It may be possible to reformulate the relativistic XMHD action principle in terms of $b^{v}$ instead of $\mathcal{F}^{\mu \nu}$. The key is how we define a generalized four vector (say $b^{\star v}$ ) that incorporates inertia effect in the similar way as $\mathcal{F}^{\mu \nu} \rightarrow \mathcal{F}^{\star \mu \nu}$. Recently, such a generalization of $b^{v}$ has been proposed by Pegoraro. ${ }^{44}$ Formulation of the action principle with $b^{\star v}$ and the unification with the MHD action principle ${ }^{29}$ will be a future work.

\section{LIMITS TO REDUCED MODELS}

In this section we show how to reduce the bracket AP to obtain APs for unknown relativistic models, with known nonrelativistic counterparts.

First consider the electron-ion plasma, where now the species labels + and - are replaced by $\mathrm{i}$ and e, respectively. Defining electron to ion mass ratio $\mu:=m_{\mathrm{e}} / m_{\mathrm{i}} \ll 1$, we approximate $m_{\mathrm{e}} / m \sim \mu, m_{\mathrm{i}} / m \sim 1$. The ion and electron fourvelocities become

$$
u_{\mathrm{i}}^{v}=u^{v}+\frac{\mu J^{v}}{n e}, \quad u_{\mathrm{e}}^{v}=u^{v}-\frac{J^{v}}{n e},
$$

while the enthalpy variables reduce to $\Delta h \sim \mu h_{\mathrm{i}}-h_{\mathrm{e}}, h^{\dagger} \sim$ $\mu^{2} h_{\mathrm{i}}+h_{\mathrm{e}}$, and $\Delta h^{\sharp} \sim \mu^{3} h_{\mathrm{i}}-h_{\mathrm{e}}$, and the generalized vectors become

$$
\begin{aligned}
& \mathrm{m}^{\star \nu}=n h u^{v}+\frac{1}{e}\left(\mu h_{\mathrm{i}}-h_{\mathrm{e}}\right) J^{v} \\
& A^{\star^{\nu}}=A^{v}+\frac{1}{e}\left(\mu h_{\mathrm{i}}-h_{\mathrm{e}}\right) u^{v}+\frac{1}{n e^{2}}\left(\mu^{2} h_{\mathrm{i}}+h_{\mathrm{e}}\right) J^{v} \\
& A^{\dagger^{\nu}}=A^{v}-\frac{1}{e}\left(\mu^{2} h_{\mathrm{i}}+h_{\mathrm{e}}\right) u^{v}-\frac{1}{n e^{2}}\left(\mu^{3} h_{\mathrm{i}}-h_{\mathrm{e}}\right) J^{\nu} .
\end{aligned}
$$

Next, in this approximation the noncanonical Poisson bracket (25) becomes

$$
\begin{aligned}
& \{\bar{F}, \bar{G}\}_{\mathrm{XMHD}}=-\int\left\{n\left(\frac{\delta \bar{G}}{\delta m^{\star \nu}} \partial^{\nu} \frac{\delta \bar{F}}{\delta n}-\frac{\delta \bar{F}}{\delta m^{\star \nu}} \partial^{\nu} \frac{\delta \bar{G}}{\delta n}\right)\right. \\
& +\mathfrak{m}^{\star \nu}\left(\frac{\delta \bar{G}}{\delta \mathfrak{m}^{\star \mu}} \partial^{\mu} \frac{\delta \bar{F}}{\delta \mathfrak{m}^{\star \nu}}-\frac{\delta \bar{F}}{\delta \mathfrak{m}^{\star \mu}} \partial^{\mu} \frac{\delta \bar{G}}{\delta \mathfrak{m}^{\star \nu}}\right) \\
& +\sigma_{\mathrm{i}}\left(\frac{\delta \bar{G}}{\delta \mathrm{m}^{\star \nu}} \partial^{\nu} \frac{\delta \bar{F}}{\delta \sigma_{\mathrm{i}}}-\frac{\delta \bar{F}}{\delta \mathrm{m}^{\star \nu}} \partial^{\nu} \frac{\delta \bar{G}}{\delta \sigma_{\mathrm{i}}}\right) \\
& -\frac{\mu}{e}\left(\frac{\delta \bar{F}}{\delta \sigma_{\mathrm{i}}} \frac{\delta \bar{G}}{\delta A^{\star \nu}}-\frac{\delta \bar{G}}{\delta \sigma_{\mathrm{i}}} \frac{\delta \bar{F}}{\delta A^{\star \nu}}\right) \partial^{v}\left(\frac{\sigma_{\mathrm{i}}}{n}\right) \\
& +\sigma_{\mathrm{e}}\left(\frac{\delta \bar{G}}{\delta m^{\star \nu}} \partial^{\nu} \frac{\delta \bar{F}}{\delta \sigma_{\mathrm{e}}}-\frac{\delta \bar{F}}{\delta m^{\star \nu}} \partial^{\nu} \frac{\delta \bar{G}}{\delta \sigma_{\mathrm{e}}}\right) \\
& +\frac{1}{e}\left(\frac{\delta \bar{F}}{\delta \sigma_{\mathrm{e}}} \frac{\delta \bar{G}}{\delta A^{\star \nu}}-\frac{\delta \bar{G}}{\delta \sigma_{\mathrm{e}}} \frac{\delta \bar{F}}{\delta A^{\star \nu}}\right) \partial^{\nu}\left(\frac{\sigma_{\mathrm{e}}}{n}\right) \\
& +\left(\frac{\delta \bar{G}}{\delta m^{\star \nu}} \frac{\delta \bar{F}}{\delta A^{\star \mu}}-\frac{\delta \bar{F}}{\delta m^{\star \nu}} \frac{\delta \bar{G}}{\delta A^{\star \mu}}\right) \mathcal{F}^{\star \mu \nu} \\
& \left.-\frac{1}{n e} \frac{\delta \bar{F}}{\delta A^{\star \mu}} \frac{\delta \bar{G}}{\delta A^{\star \nu}} \mathcal{F}^{\dagger^{\mu \nu}}\right\} \mathrm{d}^{4} x \text {. }
\end{aligned}
$$

Using the approximate bracket of (29) with a reduced action $\bar{S}$, the covariant AP produces the continuity equation (4) along 
with the following system of equations:

$$
\begin{aligned}
& \partial_{v}\left[n h u^{\mu} u^{v}+\frac{1}{e}\left(\mu h_{\mathrm{i}}-h_{\mathrm{e}}\right)\left(u^{\mu} J^{v}+J^{\mu} u^{\nu}\right)\right. \\
& \left.\quad+\frac{1}{n e^{2}}\left(\mu^{2} h_{\mathrm{i}}+h_{\mathrm{e}}\right) J^{\mu} J^{v}\right]=\partial^{\mu} p+J_{v} \mathcal{F}^{\mu \nu}, \\
& e u_{v} \mathcal{F}^{\star \mu \nu}-\frac{J_{v}}{n} \mathcal{F}^{\dagger^{\mu \nu}}-\mu T_{\mathrm{i}} \partial^{\mu}\left(\frac{\sigma_{\mathrm{i}}}{n}\right)+T_{\mathrm{e}} \partial^{\mu}\left(\frac{\sigma_{\mathrm{e}}}{n}\right)=0, \\
& \partial_{v}\left[\sigma_{\mathrm{i}}\left(u^{v}+\frac{\mu J^{v}}{n e}\right)\right]=0 \\
& \partial_{\nu}\left[\sigma_{\mathrm{e}}\left(u^{v}-\frac{J^{v}}{n e}\right)\right]=0
\end{aligned}
$$

Next consider a further reduction using $\mu \rightarrow 0$, meaning the electron rest mass inertia is discarded. This limit gives HMHD, which is well known in the nonrelativistic case but has not been proposed in the relativistic case. The terms including $h_{\mathrm{e}}$ must not be ignored when the electron thermal inertia is greater than the rest mass inertia (i.e., $h_{\mathrm{e}} \gg m_{\mathrm{e}} c^{2}$ ). For example, the temperature of electrons in an accretion disk near a black hole can be more than $10^{11} \mathrm{~K} \cdot{ }^{45}$ Then, the thermal inertia $h_{\mathrm{e}}$ is on the order of $100 m_{\mathrm{e}} c^{2}$, estimated by an equation of state for an ideal gas $h_{\mathrm{e}}=m_{\mathrm{e}} c^{2}+[\Gamma /(\Gamma-1)] T_{\mathrm{e}}$ with a specific heat ratio $\Gamma=4 / 3 .{ }^{46}$ In such a case, the $h_{\mathrm{e}}$ terms are not negligible.

Let us employ the following normalizations:

$$
\begin{array}{r}
\partial^{v} \rightarrow L^{-1} \partial^{\nu}, \quad n \rightarrow n_{0} n, \quad T_{\mathrm{i}, \mathrm{e}} \rightarrow m c^{2} T_{\mathrm{i}, \mathrm{e}}, \\
\sigma_{\mathrm{i}, \mathrm{e}} \rightarrow n_{0} \sigma_{\mathrm{i}, \mathrm{e}}, \quad \mathcal{F}^{\mu \nu} \rightarrow \sqrt{n_{0} m c^{2}} \mathcal{F}^{\mu \nu},
\end{array}
$$

using a typical scale length $L$ and density scale $n_{0}$. Then the generalized momentum density and vector potential are normalized as

$$
\begin{aligned}
& \mathrm{m}^{\star \nu} \rightarrow n_{0} m c^{2}\left[n h u^{\nu}-d_{\mathrm{i}} h_{\mathrm{e}} J^{\nu}\right] \\
& A^{\star \nu} \rightarrow L \sqrt{n_{0} m c^{2}}\left[A^{v}-d_{\mathrm{i}} h_{\mathrm{e}} u^{v}+d_{\mathrm{i}}{ }^{2} h_{\mathrm{e}} \frac{J^{\nu}}{n}\right],
\end{aligned}
$$

where $\sqrt{\left(m c^{2}\right) /\left(e^{2} n_{0} L^{2}\right)} \sim \sqrt{\left(m_{\mathrm{i}} c^{2}\right) /\left(e^{2} n_{0} L^{2}\right)}=c /\left(\omega_{\mathrm{i}} L\right)=d_{\mathrm{i}}$ is the normalized ion skin depth, and the normalized Poisson bracket becomes

$$
\begin{aligned}
\{\bar{F}, \bar{G}\}_{\mathrm{HMHD}} & =-\int\left\{n\left(\frac{\delta \bar{G}}{\delta \mathfrak{m}^{\star \nu}} \partial^{\nu} \frac{\delta \bar{F}}{\delta n}-\frac{\delta \bar{F}}{\delta \mathrm{m}^{\star \nu}} \partial^{\nu} \frac{\delta \bar{G}}{\delta n}\right)\right. \\
& +\mathrm{m}^{\star \nu}\left(\frac{\delta \bar{G}}{\delta \mathrm{m}^{\star \mu}} \partial^{\mu} \frac{\delta \bar{F}}{\delta \mathrm{m}^{\star \nu}}-\frac{\delta \bar{F}}{\delta \mathfrak{m}^{\star \mu}} \partial^{\mu} \frac{\delta \bar{G}}{\delta \mathrm{m}^{\star \nu}}\right) \\
& +\sigma_{\mathrm{i}}\left(\frac{\delta \bar{G}}{\delta \mathfrak{m}^{\star \nu}} \partial^{\nu} \frac{\delta \bar{F}}{\delta \sigma_{\mathrm{i}}}-\frac{\delta \bar{F}}{\delta \mathrm{m}^{\star \nu}} \partial^{\nu} \frac{\delta \bar{G}}{\delta \sigma_{\mathrm{i}}}\right) \\
& +\sigma_{\mathrm{e}}\left(\frac{\delta \bar{G}}{\delta \mathrm{m}^{\star \nu}} \partial^{\nu} \frac{\delta \bar{F}}{\delta \sigma_{\mathrm{e}}}-\frac{\delta \bar{F}}{\delta \mathfrak{m}^{\star \nu}} \partial^{\nu} \frac{\delta \bar{G}}{\delta \sigma_{\mathrm{e}}}\right) \\
& -2 d_{\mathrm{i}}\left(\frac{\delta \bar{F}}{\delta \sigma_{\mathrm{e}}} \partial^{\mu} \frac{\delta \bar{G}}{\delta \mathcal{F}^{\star \mu \nu}}-\frac{\delta \bar{G}}{\delta \sigma_{\mathrm{e}}} \partial^{\mu} \frac{\delta \bar{F}}{\delta F^{\star \mu \nu}}\right) \partial^{\nu} \frac{\sigma_{\mathrm{e}}}{n} \\
& +2\left(\frac{\delta \bar{F}}{\delta \mathfrak{m}^{\star \lambda}} \partial^{\mu} \frac{\delta \bar{G}}{\delta \mathcal{F}^{\star \mu \nu}}-\frac{\delta \bar{G}}{\delta m^{\star \lambda}} \partial^{\mu} \frac{\delta \bar{F}}{\delta \mathcal{F}^{\star \mu \nu}}\right) \mathcal{F}^{\star \nu \lambda} \\
& \left.+\frac{4 d_{\mathrm{i}}}{n}\left(\partial^{\mu} \frac{\delta \bar{F}}{\delta \mathcal{F}^{\star \mu \nu}}\right)\left(\partial^{\lambda} \frac{\delta \bar{G}}{\delta \mathcal{F}^{\star \lambda \kappa}}\right) \mathcal{F}^{\star \kappa \nu}\right\} \mathrm{d}^{4} x . \quad(34)
\end{aligned}
$$

The bracket AP with this scaling gives the following equations:

$$
\begin{aligned}
& \partial_{v}\left[n h u^{\mu} u^{v}-d_{\mathrm{i}} h_{\mathrm{e}}\left(u^{\mu} J^{v}+J^{\mu} u^{\nu}\right)+d_{\mathrm{i}}^{2} \frac{h_{\mathrm{e}}}{n} J^{\mu} J^{v}\right] \\
& =\partial^{\mu} p+J^{v} \mathcal{F}^{\mu}{ }_{\nu}, \\
& \left(u_{v}-d_{\mathrm{i}} \frac{J_{v}}{n}\right) \mathcal{F}^{\star \mu \nu}=-d_{\mathrm{i}} T_{\mathrm{e}} \partial^{\mu}\left(\frac{\sigma_{\mathrm{e}}}{n}\right) \\
& \partial_{v}\left(\sigma_{\mathrm{i}} u^{\nu}\right)=0, \\
& \partial_{v}\left[\sigma_{\mathrm{e}}\left(u^{v}-d_{\mathrm{i}} \frac{J^{v}}{n}\right)\right]=0 .
\end{aligned}
$$

Note, this relativistic HMHD is different from usual nonrelativistic HMHD. In Sec. V we explore some consequences of this.

Next, upon taking the limit $d_{\mathrm{i}} \rightarrow 0$, we obtain relativistic MHD. ${ }^{42,43,47}$ The Poisson bracket for the relativistic MHD obtained by this reduction is different from the one proposed by D'Avignon et al. in Ref. 29 because a magnetic field like four vector $b^{v}$ was used there instead of $A^{\mu}$. The relation between the two brackets has yet to be clarified.

The same reduction procedure (from XMHD to MHD) is applicable for the constrained least AP of Sec. II. For example, if we ignore the electron rest mass, the velocities of each species are reduced as ${u_{+}}^{\mu} \rightarrow u^{\mu}$ and $u_{-}{ }^{\mu} \rightarrow u^{\mu}-J^{\mu} / n e$. Similarly, the entropy and Lagrangian label constraints are reduced accordingly. With these reductions the constrained least AP gives the relativistic HMHD equations. We note, the renormalization method used in Ref. 19 to derive AP for MHD is also applicable for relativistic HMHD.

There are formalisms alternative to the one we presented that employ either Ohm's law or the induction equation per se as a constraint for nonrelativistic ${ }^{25}$ and relativistic ${ }^{26,27}$ MHD. However, these formulations cannot be reduced from the constrained action (7). Whereas the physical meaning of the constraints in (7) is obvious, embedding the Ohm's law as a constraint is unnatural and arbitrary. Furthermore, the EM field cannot be expressed by Clebsch potentials from the AP with 
the Ohm's law constraint, unlike the case for our formulation where this emerges naturally in (9).

\section{RELATIVISTIC COLLISIONLESS RECONNECTION}

In nonrelativistic MHD with the inclusion of electron (rest mass) inertia (i.e., IMHD), a consequence of electron inertia is the violation of the frozen-in magnetic flux condition, and instead, a flux determined by a generalized field is conserved. ${ }^{10}$ Such an electron inertia effect was suggested as a mechanism for a collisionless magnetic reconnection ${ }^{48}$ and has now been widely studied. However, nonrelativistic HMHD does satisfy the frozen-in magnetic flux condition because the electron inertia is discarded by the $\mu \rightarrow 0$ limit. Hence, there is no direct mechanism causing collisionless reconnection in nonrelativistic HMHD.

On the other hand, in relativistic XMHD, there are two kinds of electron inertiae: one from the electron rest mass $m_{\mathrm{e}}$ and the other from the electron temperature $h_{\mathrm{e}}$. The $\mu \rightarrow 0$ limit corresponds to neglecting the former and keeping the latter. Even though the former is small, the latter may not be ignorable when electron temperature is large enough. The latter effect still allows for the violation of the frozen-in magnetic flux condition. Such a collisionless reconnection mechanism was previously proposed by Comisso et al. using a SweetParker model in the context of relativistic XMHD. ${ }^{34}$ Here we find an alternative flux given by the generalized vector potential $A^{\star \nu} \rightarrow A^{\nu}-d_{\mathrm{i}} h_{\mathrm{e}} u^{\nu}+d_{\mathrm{i}}^{2}\left(h_{\mathrm{e}} / n\right) J^{\nu}$ to be frozen-in.

Let us stress the difference between our present study and the pair plasma study by Comisso et al. ${ }^{34}$ In the latter, the relativistic electron-positron plasma with the assumption $\Delta h=0$ was considered. For HMHD, however, this $\Delta h=0$ assumption removes the aforementioned collisionless reconnection mechanism. From (27) and (28), we find $A^{\star \mu} \rightarrow A^{\mu}$ and $A^{\dagger \mu} \rightarrow A^{\mu}$ when we take both $\Delta h=0$ and $\mu=0$, so there is no longer the alternative frozen-in flux in HMHD.

To make this statement more explicit, we write the relativistic HMHD induction equation in a reference frame moving with the center-of-mass (ion) velocity. When the electron fluid is homentropic, the right-hand side of (36) vanishes. Taking a curl of a spatial component of (36), we obtain the induction equation in the reference frame,

$$
\partial_{t} \mathbf{B}^{\star}+\nabla \times\left(\mathbf{B}^{\star} \times \widetilde{\mathbf{v}}_{\mathrm{e}}\right)=0,
$$

where

$$
\mathbf{B}^{\star}=\mathbf{B}+\nabla \times\left(-d_{\mathrm{i}} h_{\mathrm{e}} \gamma \mathbf{v}+d_{\mathrm{i}}^{2} h_{\mathrm{e}} \frac{\mathbf{J}}{n}\right)
$$

and

$$
\widetilde{\mathbf{v}}_{\mathrm{e}}=\left(\mathbf{v}-d_{\mathrm{i}} \frac{\mathbf{J}}{\gamma n}\right)\left(1-d_{\mathrm{i}} \frac{\rho_{q}}{\gamma n}\right)^{-1} .
$$

Here, $\widetilde{\mathbf{v}}_{\mathrm{e}}$ is a modified electron velocity that becomes the electron velocity $\mathbf{v}_{\mathrm{e}}$ in the nonrelativistic limit $\gamma \rightarrow 1$ and $\rho_{q} \rightarrow 0$. Evidently from (39) and (40), the magnetic field $\mathbf{B}$ is no longer frozen-in.
Let us compare (40) with the induction equations for other magnetohydrodynamic models, summarized in Table II. The frozen-in condition for $\mathbf{B}$ is satisfied in nonrelativistic MHD, HMHD, and relativistic MHD. The frozen-in condition is violated in nonrelativistic two-dimensional IMHD, while the alternative field $\mathbf{B}+\nabla \times\left(d_{\mathrm{e}}^{2} \mathbf{J} / n\right)$, with the electron skin depth $d_{\mathrm{e}}$ as characteristic length ${ }^{48}$ is frozen-in. Therefore, the scale length of the collisionless reconnection caused by the electron inertia is $d_{\mathrm{e}}$. On the other hand, the alternative frozen-in field in relativistic HMHD is $\mathbf{B}+\nabla \times\left(-d_{\mathrm{i}} h_{\mathrm{e}} \gamma \mathbf{v}+d_{\mathrm{i}}^{2} h_{\mathrm{e}} \mathbf{J} / n\right)$, which has a characteristic scale length with $\sqrt{h_{\mathrm{e}}} d_{\mathrm{i}}$. Since the scale length $d_{\mathrm{e}}$ in nonrelativistic IMHD is replaced to $\sqrt{h_{\mathrm{e}}} d_{\mathrm{i}}$ in relativistic HMHD, and the reconnection scale is expected to be $\sqrt{h_{\mathrm{e}}} d_{\mathrm{i}}$. This estimate is the same as that for the Sweet-Parker model for relativistic electron-positron $\mathrm{XMHD}^{34}$ (recall that $h_{\mathrm{e}}$ is normalized by $m c^{2}$ in this study).

Here we have inferred the reconnection scale just by comparing non-relativistic and relativistic Ohm's law. However, in non-relativistic case, it was shown that the reconnection scale is not determined by the generalized Ohm's law alone when there is a strong magnetic guide field and appropriate gyro-physics is added to the model. The analysis of the resulting gyrofluid model revealed that the relevant scale becomes the ion sound Larmor radius in this case. ${ }^{49}$ Inclusion of gyroscopic effects in the relativistic context, appropriate for strong guide fields, is a subject for future work.

\section{NONRELATIVISTIC XMHD - 3+1 DECOMPOSITION}

The covariant Poisson bracket AP formalism also encompasses nonrelativistic theories. We will show this in the context of XMHD, then infer that this is the case for nonrelativistic MHD and the nonrelativistic ideal fluid. Because nonrelativistic theories contain space and time separately, it is natural to pursue this end by beginning from the $3+1$ decomposition for relativistic theories described in Ref. 28. To this end we state some general tools before proceeding to the task at hand.

The functional derivative of (22) is defined relative to the space-time pairing, while functional derivatives in conventional Hamiltonian theories are defined relative to only the spatial pairing, i.e.

$$
\delta \mathscr{F}=\int \delta n \frac{\delta \mathscr{F}}{\delta n} \mathrm{~d}^{3} x
$$

For functionals of the form $F=\int \mathscr{F} \mathrm{d} x^{0}$ where $\mathscr{F}$ contains no time derivatives of a field, it follows e.g. that

$$
\frac{\delta F}{\delta n\left(x^{0}, \mathbf{x}\right)}=\frac{\delta \mathscr{F}}{\delta n(\mathbf{x})}
$$

where we explicitly display the arguments to distinguish space-time from space functional derivatives. For nonrelativistic theories, we need to consider functionals that are localized in time, i.e., have the form

$$
F=\int \delta\left(x^{0}-x^{0^{\prime}}\right) \mathscr{F} \mathrm{d} x^{0} .
$$


TABLE II. Induction equations for nonrelativistic MHD, HMHD and IMHD, and relativistic MHD and HMHD.

\begin{tabular}{|r|c|c|}
\hline & Barotropic induction eq. & frozen-in field \\
\hline Nonrelativistic MHD & $\partial_{t} \mathbf{B}+\nabla \times(\mathbf{B} \times \mathbf{v})=0$ & $\mathbf{B}$ \\
\hline Nonrelativistic HMHD & $\partial_{t} \mathbf{B}+\nabla \times\left(\mathbf{B} \times \mathbf{v}_{\mathrm{e}}\right)=0$ & $\mathbf{B}$ \\
\hline Nonrelativistic 2D IMHD & $\partial_{t} \mathbf{B}^{\star}+\nabla \times\left(\mathbf{B}^{\star} \times \mathbf{v}\right)=0$ & $\mathbf{B}^{\star}=\mathbf{B}+\nabla \times\left(d_{\mathrm{e}}^{2} \mathbf{J} / n\right)$ \\
\hline Relativistic MHD & $\partial_{t} \mathbf{B}+\nabla \times(\mathbf{B} \times \mathbf{v})=0$ & $\mathbf{B}$ \\
\hline Relativistic HMHD & $\partial_{t} \mathbf{B}^{\star}+\nabla \times\left(\mathbf{B}^{\star} \times \widetilde{\mathbf{v}}_{\mathrm{e}}\right)=0$ & $\mathbf{B}^{\star}=\mathbf{B}+\nabla \times\left(-d_{\mathrm{i}} h_{\mathrm{e}} \gamma \mathbf{v}+d_{\mathrm{i}}^{2} h_{\mathrm{e}} \mathbf{J} / n\right)$ \\
\hline
\end{tabular}

Observe, in this case, if $\mathscr{F}$ contains no time derivatives of the field $n$, then

$$
\frac{\delta F}{\delta n\left(x^{0}, \mathbf{x}\right)}=\delta\left(x^{0}-x^{0^{\prime}}\right) \frac{\delta \mathscr{F}}{\delta n(\mathbf{x})},
$$

and similarly for other fields. Next, let us suppose that a functional $G$ is separable in the following sense

$$
G=G^{0}+\int \mathscr{G} \mathrm{d} x^{0}
$$

where all of the time-like components of fields are contained in the functionals $G^{0}$ and $\mathscr{G}$ contains no time derivatives of fields. For functionals $G$ of the form of (46) and $F$ of the form of (44), it will be shown that

$$
0=\{F, G\}=-\frac{\mathrm{d} \mathscr{F}}{\mathrm{d} t}+\{\mathscr{F}, \mathscr{G}\}^{(3)}
$$

where $\{F, G\}$ is the canonical bracket (21) or the noncanonical bracket (23), and $\{\mathscr{F}, \mathscr{G}\}^{(3)}$ is the appropriate nonrelativistic Poisson bracket. In this way one can establish the connection between Poisson bracket APs and usual noncanonical Poisson bracket Hamiltonian formulations.

For the case at hand, let us return to the arbitrary mass plasma $\left(m_{+}\right.$and $\left.m_{-}\right)$and consider a nonrelativistic limit with

$h \rightarrow m c^{2}, \quad \Delta h \rightarrow 0, \quad h^{\dagger} \rightarrow\left(m_{+} m_{-}\right) c^{2} / m, \quad \gamma_{ \pm} \rightarrow 1, \partial_{t} \mathbf{E}=0$.

These result in $J^{0}=e n\left(\gamma_{+}-\gamma_{-}\right) \rightarrow 0$ and $\mathbf{J}=\nabla \times \mathbf{B}$, and the generalized fields become

$$
\begin{aligned}
& \mathrm{m}^{\star v} \rightarrow n m c^{2} u^{v}=\mathrm{m}^{v}, \\
& A^{\star v} \rightarrow A^{v}+\frac{1}{n e^{2}}\left(\frac{m_{+} m_{-}}{m} c^{2}\right) J^{v},
\end{aligned}
$$

and

$$
A^{\dagger v}=\frac{m_{+}-m_{-}}{m} A^{\star v}-\frac{m_{-} m_{+} c}{m e} u^{v},
$$

with the four-velocity becoming $u^{v}=(1, \mathbf{v} / c)$. Using the thermodynamic relations $\rho=n\left(m c^{2}+\mathcal{E}\right)$ and $p=n h-\rho$, with internal energy $\mathcal{E}$, the following limit is calculated

$$
\frac{1}{2}(\rho-p)=n\left(m c^{2}+\mathcal{E}\right)-\frac{1}{2} n h \rightarrow n\left(\frac{1}{2} m c^{2}+\mathcal{E}\right) .
$$

We first show a nonrelativistic Hamilton's equation for the Clebsch variables. The action (7) is separated as

$$
S[z]=\int\left[n \partial^{0} \phi+\sum_{ \pm}\left(\sigma_{ \pm} \partial^{0} \eta_{ \pm}+\varphi_{ \pm} \partial^{0} \lambda_{ \pm}\right)\right] \mathrm{d}^{4} x-\int \mathscr{H} \mathrm{d}^{0} x
$$

with a Hamiltonian

$$
\mathscr{H}[z]=\int\left[n\left(m c^{2}+\mathcal{E}_{+}+\mathcal{E}_{-}\right)+\frac{1}{2} n m v^{2}+\frac{1}{2} \mathbf{J} \cdot \mathbf{A}^{\star}\right] \mathrm{d}^{3} x .
$$

Here $\mathbf{v}=\mathbf{m} / n m c, \mathbf{A}^{\star}$, and $\mathbf{J}$ are functions of $z$. Substituting, this action and the localized functional (44) into the covariant canonical bracket (21), we get

$$
\{F, S\}_{\text {canonical }}=\int\left(\frac{\mathrm{d} \mathscr{F}}{\mathrm{d} t}-\{\mathscr{F}, \mathscr{H}\}_{\text {canonical }}^{(3)}\right) \delta\left(x^{0}-x^{0^{\prime}}\right) \mathrm{d} x^{0}
$$

where $\{\mathscr{F}, \mathscr{H}\}_{\text {canonical }}^{(3)}$ is a canonical Poisson bracket defined in the three-dimensional space. Thus, we get the nonrelativistic canonical Hamilton's equation as

$$
\frac{\mathrm{d} \mathscr{F}}{\mathrm{d} t}=\{\mathscr{F}, \mathscr{H}\}_{\text {canonical }}^{(3)},
$$

which describes the time evolution of the Clebsch variables z. Transforming the Clebsch variables to $\mathbf{v}$ and $\mathbf{B}^{\star}$, we obtain the non-relativistic XMHD equations, which will be explicitly shown below.

Now we are set to apply this $3+1$ procedure to the noncanonical bracket (23). Upon rearranging the action of (20) we obtain

$$
\bar{S}=\int \frac{m_{0} m^{0}}{2 n m c^{2}} \mathrm{~d}^{4} x-\int \mathscr{H} \mathrm{d} x^{0},
$$

with the Hamiltonian

$$
\begin{aligned}
& \mathscr{H}\left[n, \sigma, \mathrm{m}^{i}, A^{\star i}\right]= \\
& \int\left[-\frac{\mathrm{m}_{i} \mathrm{~m}^{i}}{2 n m c^{2}}+n\left(\frac{1}{2} m c^{2}+\mathcal{E}_{+}+\mathcal{E}_{-}\right)-\frac{A^{\star} J_{i}}{2}\right] \mathrm{d}^{3} x,
\end{aligned}
$$

where we used $J^{0}=0$ to get the last term.

Then we calculate $\{\bar{F}, \bar{S}\}_{\mathrm{XMHD}}=0$ to get the nonrelativistic XMHD equations. The phase space variables must be $\left(n, \sigma_{ \pm}, \mathrm{m}^{i}, A^{\star i}\right)$. Hence we put $\bar{F}=\bar{F}\left[n, \sigma_{ \pm}, \mathrm{m}^{i}, A^{\star i}\right]$. Since the action (49) does not depends on $A^{\star 0}$, we may write $\bar{S}=\bar{S}\left[n, \sigma_{ \pm}, \mathrm{m}^{0}, \mathrm{~m}^{i}, A^{\star i}\right]$. Therefore all the terms including $\delta \bar{F} / \delta \mathrm{m}^{0}, \delta \bar{F} / \delta A^{\star 0}$, and $\delta \bar{S} / \delta A^{\star 0}$ are dropped. Upon writing

$$
\bar{F}=\int \delta\left(x^{0}-x^{0^{\prime}}\right) \mathscr{F}\left[n, \sigma_{ \pm}, \mathrm{m}^{i}, A^{\star i}\right] \mathrm{d} x^{0},
$$


the covariant bracket AP can be written as

$$
\begin{aligned}
& 0=\{\bar{F}, \bar{S}\}_{\mathrm{XMHD}}=-\int\left\{n \frac{\mathrm{m}_{0}}{n m c^{2}} \partial^{0}\left[\delta\left(x^{0}-x^{0^{\prime}}\right) \frac{\delta \mathscr{F}}{\delta n}\right]\right. \\
& +\delta\left(x^{0}-x^{0^{\prime}}\right) n\left[-\frac{\delta \mathscr{H}}{\delta \mathrm{m}^{i}} \partial^{i} \frac{\delta \mathscr{F}}{\delta n}+\frac{\delta \mathscr{F}}{\delta \mathrm{m}^{i}} \partial^{i}\left(\frac{\mathrm{m}_{0} \mathrm{~m}^{0}}{2 n^{2} m c^{2}}+\frac{\delta \mathscr{H}}{\delta n}\right)\right] \\
& +\sum_{ \pm}\left(\sigma_{ \pm} \frac{m_{0}}{n m c^{2}} \partial^{0}\left[\delta\left(x^{0}-x^{0^{\prime}}\right) \frac{\delta \mathscr{F}}{\delta \sigma_{ \pm}}\right]\right. \\
& +\delta\left(x^{0}-x^{0^{\prime}}\right) \sigma_{ \pm}\left[-\frac{\delta \mathscr{H}}{\delta m^{i}} \partial^{i} \frac{\delta \mathscr{F}}{\delta \sigma_{ \pm}}+\frac{\delta \mathscr{F}}{\delta m^{i}} \partial^{i}\left(\frac{\delta \mathscr{H}}{\delta \sigma_{ \pm}}\right)\right] \\
& \left. \pm \delta\left(x^{0}-x^{0^{\prime}}\right) \frac{m_{\mp}}{m e}\left(\frac{\delta \mathscr{F}}{\delta \sigma_{ \pm}} \frac{\delta \mathscr{H}}{\delta A^{\star} i}-\frac{\delta \mathscr{H}}{\delta \sigma_{ \pm}} \frac{\delta \mathscr{F}}{\delta A^{\star}}\right) \partial^{i}\left(\frac{\sigma_{ \pm}}{n}\right)\right) \\
& +\mathrm{m}^{i}\left(\frac{\mathrm{m}^{0}}{n m c^{2}} \partial^{0}\left[\delta\left(x^{0}-x^{0^{\prime}}\right) \frac{\delta \mathscr{F}}{\delta \mathrm{m}^{i}}\right]\right)+\delta\left(x^{0}-x^{0^{\prime}}\right) \mathrm{m}^{0}\left(-\frac{\delta \mathscr{F}}{\delta \mathrm{m}^{i}} \partial^{i} \frac{\mathrm{m}^{0}}{n m c^{2}}\right) \\
& -\delta\left(x^{0}-x^{0^{\prime}}\right) \mathrm{m}^{j}\left(\frac{\delta \mathscr{H}}{\delta \mathrm{m}^{i}} \partial^{i} \frac{\delta \mathscr{F}}{\delta \mathrm{m}^{j}}-\frac{\delta \mathscr{F}}{\delta \mathrm{m}^{i}} \partial^{i} \frac{\delta \mathscr{H}}{\delta \mathrm{m}^{j}}\right) \\
& +\delta\left(x^{0}-x^{0^{\prime}}\right)\left(\frac{\mathrm{m}_{0}}{n m c^{2}} \frac{\delta \mathscr{F}}{\delta A^{\star i}}\right)\left(\partial^{i} A^{\star 0}-\partial^{0} A^{\star i}\right) \\
& +\delta\left(x^{0}-x^{0^{\prime}}\right)\left(\frac{\delta \mathscr{H}}{\delta \mathrm{m}^{i}} \frac{\delta \mathscr{F}}{\delta A^{\star} j}-\frac{\delta \mathscr{F}}{\delta \mathrm{m}^{i}} \frac{\delta \mathscr{H}}{\delta A^{\star} j}\right) F^{\star j i} \\
& \left.-\delta\left(x^{0}-x^{0^{\prime}}\right) \frac{1}{n e} \frac{\delta \mathscr{F}}{\delta A^{\star}} \frac{\delta \mathscr{F}}{\delta A^{\star j}} F^{\dagger^{i j}}\right\} \mathrm{d}^{4} x \text {. }
\end{aligned}
$$

Next we substitute $m^{0}=m_{0}=n m c^{2}$ and manipulate some of the terms to obtain

$$
\begin{aligned}
\int\left(\frac{\delta \mathscr{F}}{\delta n} \partial^{0} n\right. & +\frac{\delta \mathscr{F}}{\delta \sigma_{+}} \partial^{0} \sigma_{+}+\frac{\delta \mathscr{F}}{\delta \sigma_{-}} \partial^{0} \sigma_{-} \\
& \left.+\frac{\delta \mathscr{F}}{\delta \mathrm{m}^{i}} \partial^{0} \mathrm{~m}^{i}+\frac{\delta \mathscr{F}}{\delta A^{\star} i} \partial^{0} A^{\star i}\right) \mathrm{d}^{3} x=\frac{1}{c} \frac{\mathrm{d} \mathscr{F}}{\mathrm{d} t}
\end{aligned}
$$

yielding

$$
\begin{aligned}
\{\bar{F}, \bar{S}\}_{\mathrm{XMHD}}=\frac{1}{c} \int & \left(\frac{\mathrm{d} \mathscr{F}}{\mathrm{d} t}-\{\mathscr{F}, \mathscr{H}\}^{(3)}\right) \delta\left(x^{0}-x^{0^{\prime}}\right) \mathrm{d} x^{0} \\
& +\int A^{\star 0} \partial^{i}\left(\frac{\delta \mathscr{F}}{\delta A^{\star}}\right) \delta\left(x^{0}-x^{0^{\prime}}\right) \mathrm{d}^{4} x
\end{aligned}
$$

with a three dimensional Poisson bracket $\{\mathscr{F}, \mathscr{G}\}^{(3)}$ that will be explicitly shown below. Evaluating the $\delta$-function shows $\{\bar{F}, \bar{S}\}_{\mathrm{XMHD}}=0$ is equivalent to Hamilton's equation along with a gauge-like condition:

$$
\frac{\mathrm{d} \mathscr{F}}{\mathrm{d} t}=\{\mathscr{F}, \mathscr{H}\}^{(3)} \quad \text { and } \quad \nabla \cdot\left(\frac{\delta \mathscr{F}}{\delta \mathbf{A}^{\star}}\right)=0 .
$$

The second equation of (51), the gauge condition, is handled manifestly by transforming from the phase space variable $\mathbf{A}^{\star}$ to $\mathbf{B}^{\star}$; since $\delta \mathscr{F} / \delta \mathbf{A}^{\star}=\nabla \times\left(\delta \mathscr{F} / \delta \mathbf{B}^{\star}\right)$, with this transformation the second condition is automatically satisfied. Finally, we transform $\mathbf{m}$ to $\mathbf{v}$ and find that the Poisson bracket
$\{\mathscr{F}, \mathscr{G}\}^{(3)}$ becomes

$$
\begin{aligned}
\{\mathscr{F}, \mathscr{G}\}^{(3)}= & \int\left\{\left(\frac{\delta \mathscr{G}}{\delta \mathbf{v}} \cdot \nabla \frac{\delta \mathscr{F}}{\delta \varrho}-\frac{\delta \mathscr{F}}{\delta \mathbf{v}} \cdot \nabla \frac{\delta \mathscr{G}}{\delta \varrho}\right)\right. \\
& +\frac{\nabla \times \mathbf{v}}{\varrho} \cdot\left(\frac{\delta \mathscr{F}}{\delta \mathbf{v}} \times \frac{\delta \mathscr{G}}{\delta \mathbf{v}}\right) \\
& +\sum_{ \pm}\left[\frac{\sigma_{ \pm}}{\varrho}\left(\frac{\delta \mathscr{G}}{\delta \mathbf{v}} \cdot \nabla \frac{\delta \mathscr{F}}{\delta \sigma_{ \pm}}-\frac{\delta \mathscr{F}}{\delta \mathbf{v}} \cdot \nabla \frac{\delta \mathscr{G}}{\delta \sigma_{ \pm}}\right)\right. \\
& \mp \frac{c m_{\mp}}{e}\left(\frac{\delta \mathscr{F}}{\delta \sigma_{ \pm}}\left(\nabla \times \frac{\delta \mathscr{G}}{\delta \mathbf{B}^{\star}}\right)\right. \\
& -\left[\frac{\delta \mathscr{G}}{\delta \mathbf{v}} \times\left(\nabla \times \frac{\delta \mathscr{F}}{\delta \mathbf{B}^{\star}}\right)-\frac{\delta \mathscr{F}}{\delta \mathbf{v}} \times\left(\nabla \times \frac{\delta \mathscr{G}}{\delta \mathbf{B}^{\star}}\right)\right] \cdot \frac{\mathbf{B}^{\star}}{\varrho} \\
& \left.\left.-\frac{m c}{\varrho e}\left[\left(\nabla \times \frac{\delta \mathscr{F}}{\delta \mathbf{B}^{\star}}\right) \times\left(\nabla \times \frac{\delta \mathscr{G}}{\delta \mathbf{B}^{\star}}\right)\right] \cdot \mathbf{B}^{\dagger}\right\} \mathrm{d}^{3} x, \quad(52) \cdot \nabla\left(\frac{\sigma_{ \pm}}{\varrho}\right)\right]
\end{aligned}
$$

where $\varrho=m n$ and $\nabla=-\partial^{i}$. This Poisson bracket is a generalization of the nonrelativistic electron-ion XMHD bracket proposed before ${ }^{37,38}$. The bracket of (52) differs from the previous results by the choice of scaling and, more importantly, the assumption $m_{-} \ll m_{+}$is not made.

Now consider the Hamiltonian of (50); it becomes

$$
\begin{aligned}
& \mathscr{H}\left[n, \sigma, \mathbf{v}, \mathbf{B}^{\star}\right]= \\
& \quad \int\left[\frac{\varrho|\mathbf{v}|^{2}}{2}+\varrho\left(\frac{1}{2} m c^{2}+\mathcal{E}_{+}+\mathcal{E}_{-}\right)+\frac{\mathbf{B}^{\star} \cdot \mathbf{B}}{2}\right] \mathrm{d}^{3} x,
\end{aligned}
$$

where $\mathcal{E}_{ \pm} / m$ is rewritten as $\mathcal{E}_{ \pm}$. The functional derivatives of $\mathscr{H}$ are

$$
\begin{aligned}
& \frac{\delta \mathscr{H}}{\delta \varrho}=\frac{1}{m} \frac{\delta \mathscr{H}}{\delta n}=\frac{v^{2}}{2}+\frac{c^{2}}{2}+\sum_{ \pm}\left(\mathcal{E}_{ \pm}+\varrho \frac{\partial \mathcal{E}_{ \pm}}{\partial \varrho}\right)+\frac{m_{+} m_{-} c^{2}}{2 \varrho^{2} e^{2}} J^{2}, \\
& \frac{\delta \mathscr{H}}{\delta \sigma_{ \pm}}=\varrho \frac{\partial \mathcal{E}_{ \pm}}{\partial \sigma_{ \pm}}, \quad \frac{\delta \mathscr{H}}{\delta \mathbf{v}}=\varrho \mathbf{v}, \quad \frac{\delta \mathscr{H}}{\delta \mathbf{B}^{\star}}=\mathbf{B} .
\end{aligned}
$$

Finally, using the above Hamilton's equations of (51) give

$$
\begin{aligned}
& \frac{\partial \varrho}{\partial t}=\{\varrho, \mathscr{H}\}^{(3)}=-\nabla \cdot(\varrho \mathbf{v}) \\
& \frac{\partial \sigma_{ \pm}}{\partial t}=\left\{\sigma_{ \pm}, \mathscr{H}\right\}^{(3)}=-\nabla \cdot\left[\left(\mathbf{v} \pm \frac{c m_{\mp}}{\varrho e} \mathbf{J}\right) \sigma_{ \pm}\right] \\
& \frac{\partial \mathbf{B}^{\star}}{\partial t}=\left\{\mathbf{B}^{\star}, \mathscr{H}\right\}^{(3)}=\sum \pm \nabla \times\left[\frac{c m_{\mp}}{e} T_{ \pm} \nabla\left(\frac{\sigma_{\mp}}{\varrho}\right)\right] \\
&+\nabla \times\left(\mathbf{v} \times \mathbf{B}^{\star}\right)-\nabla \times\left(\frac{m c}{\varrho e} \mathbf{J} \times \mathbf{B}^{\dagger}\right) \\
& \frac{\partial \mathbf{v}}{\partial t}=\{\mathbf{v}, \mathscr{H}\}^{(3)}=-(\nabla \times \mathbf{v}) \times \mathbf{v}-\nabla\left(\frac{v^{2}}{2}+\frac{m_{+} m_{-} c^{2}}{2 \varrho^{2} e^{2}} J^{2}\right)-\frac{\nabla p}{\varrho}+\frac{\mathbf{J} \times \mathbf{B}^{\star}}{\varrho},
\end{aligned}
$$

the nonrelativistic Lüst equations ${ }^{50}$. Note, here we used the thermodynamic relations

$$
\mathrm{d} \mathcal{E}=T \mathrm{~d}\left(\frac{\sigma}{\varrho}\right)+\frac{p}{\varrho^{2}} \mathrm{~d} \varrho=\frac{T}{\varrho} \mathrm{d} \sigma+\frac{1}{\varrho^{2}}(p-T \sigma) \mathrm{d} \varrho
$$


and

$$
\varrho \mathrm{d}\left(\mathcal{E}+\varrho \frac{\partial \mathcal{E}}{\partial \varrho}\right)+\sigma \mathrm{d}\left(\varrho \frac{\partial \mathcal{E}}{\partial \sigma}\right)=\mathrm{d} p
$$

In closing this section, we seek the Casimirs of (52) for the barotropic case. They must satisfy $\forall F: 0=\{F, C\}$ leading to a system

$$
\begin{gathered}
\nabla \times\left(\frac{\mathbf{B}^{\star}}{\varrho} \times C_{\mathbf{v}}+C_{\mathbf{A}^{\star}} \times \frac{\mathbf{B}^{\star}}{\varrho}\right)=0 \\
\nabla \cdot C_{\mathbf{v}}=0 \quad \text { and } \quad C_{\mathbf{v}} \times \frac{\nabla \times \mathbf{v}}{\varrho}+C_{\mathbf{A}^{\star}} \times \frac{\mathbf{B}^{\star}}{\varrho}-\nabla C_{\varrho}=0,
\end{gathered}
$$

where we use the abbreviated notation $C_{\varrho}:=\delta C / \delta \varrho$. Seeking a helicity Casimir we assume a linear combination

$$
C^{(\lambda)}=\frac{1}{2} \int d^{3} x\left(\mathbf{A}^{\star}+\lambda \mathbf{v}\right) \cdot\left(\mathbf{B}^{\star}+\lambda \nabla \times \mathbf{v}\right),
$$

which is substituted into (54) and (55) leading to a quadratic equation for $\lambda$ with roots $\lambda_{ \pm}= \pm m_{ \pm} c / e$. These new Casimirs constitute topological constraints for a plasma with $m_{+}, m_{-}$ species masses. In the limit $m_{-} \ll m_{+}$these Casimirs become those of Refs. 37 and 38. For a discussion of topological properties of XMHD see Ref. ${ }^{51}$. Notice that the $C^{ \pm}$coincide exactly with the known 2-fluid canonical helicities $\int P \wedge d P$ for each species of Refs. 33 and 52. However we emphasize here the importance of the variables $\mathbf{v}$ and $\mathbf{A}^{\star}$.

In addition the helicity Casimirs, when barotropic condition is violated we obtain the family

$$
C^{(\sigma)}=\int \mathrm{d}^{3} x \varrho f\left(\frac{\sigma_{+}}{\varrho}, \frac{\sigma_{-}}{\varrho}\right),
$$

albeit with the condition $\sigma_{+} / \varrho$ being a function of $\sigma_{-} / \varrho$ or $f_{\text {,+- }}=0$, where $f_{,+}$denotes differentiation with respect to the first argument.

\section{CONCLUSION}

We have formulated APs for relativistic XMHD. For the constrained least action principle, the constraints, namely, conservation of number density, entropy, and Lagrangian labels for each species, were employed in the manner of Lin. Extremization of the constrained action led to Clebsch potential expressions for the generalized momentum and the generalized vector potential. Then, variable transformation from the Clebsch potentials to the physical variables led to the covariant Poisson bracket for XMHD. In the Poisson bracket AP the constraints are hidden in the degeneracy of the Poisson bracket. Through these APs we have unified the Eulerian APs for all magnetofluid models. Indeed, returning to Table I we see that all slots for Eulerian APs have been completed. Now, the only remaining work is the formulation of the AP for relativistic XMHD in the Lagrangian description. Examination of the results of Ref. 11 for nonrelativistic XMHD suggests this may not be an easy task.
Another important result was our formulation of relativistic HMHD, obtained by taking a limit of the AP for XMHD. We observed that while nonrelativistic HMHD does not have a direct mechanism for collisionless reconnection, relativistic HMHD does allow the violation of the frozen-in magnetic flux condition via the electron thermal inertia effect. We also found an alternative frozen-in flux, in a manner similar to that for nonrelativistic IMHD. The scale length of the collisionless reconnection was shown to correspond to the reconnection layer width estimated by the Sweet-Parker model. ${ }^{34}$ Further study of relativistic HMHD, such as a numerical simulation of (39), will be the subject of future work.

Lastly in this paper, we passed to a nonrelativistic limit within the covariant bracket formalism, thus arriving at a "covariant" bracket for nonrelativistic XMHD. Then we derived the usual $3+1$ noncanonical Poisson bracket. However, beyond the results of Refs. 37 and 51, the result of (52) does not assume smallness of electron mass and thus is also applicable to electron-positron plasmas.

\section{ACKNOWLEDGMENTS}

The work of YK was supported by JSPS KAKENHI Grant Number 26800279. GM and PJM were supported by U.S. Dept. of Energy under contract \#DE-FG02-04ER-54742. PJM would also like to acknowledge support from the Alexander von Humboldt Foundation and the hospitality of the Numerical Plasma Physics Division of the IPP, Max Planck, Garching.

${ }^{1}$ E. Noether, Nachr. d. Konig. Gesellsch. d. Wiss. zu Gottingen, Math-phys. Klasse 1918, 235 (1918).

${ }^{2}$ D. Pfirsch and P. J. Morrison, Phys. Rev. A 32, 1714 (1985).

${ }^{3}$ J. R. Cary and A. J. Brizard, Rev. Mod. Phys. 81, 693 (2009).

${ }^{4}$ I. Keramidas Charidakos, M. Lingam, P. J. Morrison, R. L. White, and A. Wurm, Physics of Plasmas 21, 092118 (2014).

${ }^{5}$ E. G. Evstatiev and B. A. Shadwick, Journal of Computational Physics 245, 376 (2013).

${ }^{6}$ J. Xiao, H. Qin, P. J. Morrison, J. Liu, Z. Yu, R. Zhang, and Y. He, Phys. Plasmas 23, 112107 (2016).

${ }^{7}$ M. Kraus, K. Kormann, P. J. Morrison, and E. Sonnendrücker, "GEMPIC: Geometric electromagnetic particle-in-cell methods," (2016), arXiv:1609.03053v1 [math.NA], 1609.03053.

${ }^{8}$ J. L. Lagrange, Mécanique Analitique (Chez la Veuve Desaint, 1788).

${ }^{9}$ W. A. Newcomb, Nuclear Fusion: Supplement, part 2, 451 (1962).

${ }^{10} \mathrm{M}$. Lingam, P. J. Morrison, and E. Tassi, Physics Letters A 379, 570 (2015).

${ }^{11}$ E. C. D'Avignon, P. J. Morrison, and M. Lingam, Physics of Plasmas 23, 062101 (2016).

${ }^{12}$ A. H. Taub, Physical Review 94, 1468 (1954).

${ }^{13}$ R. Dewar, Australian Journal of Physics 30, 533 (1977).

${ }^{14}$ R. Salmon, Geophysical \& Astrophysical Fluid Dynamics 43, 167 (1988).

${ }^{15}$ A. Achterberg, Physical Review A 28, 2449 (1983).

${ }^{16}$ Y. Kawazura, Z. Yoshida, and Y. Fukumoto, Journal of Physics A: Mathematical and Theoretical 47, 465501 (2014).

${ }^{17}$ J. Serrin, in Encyclopedia of Physics, Vol. VIII (Springer-Verlag, 1959) pp. 125-263.

${ }^{18}$ C. C. Lin, in Proceedings of International School Physics Enrico Fermi XXI (Academic Press, 1963) p. 93.

${ }^{19}$ Z. Yoshida and E. Hameiri, Journal of Physics A: Mathematical and Theoretical 46, 335502

${ }^{20}$ B. F. Schutz, Physical Review D 2, 2762 (1970).

${ }^{21}$ J. R. Ray, Journal of Mathematical Physics 13, 1451 (1972).

${ }^{22}$ B. F. Schutz and R. Sorkin, Annals of Physics 107, 1 (1977). 
${ }^{23}$ G. Kentwell, Physics Letters A 108, 248 (1985).

${ }^{24}$ H.-T. Elze, Y. Hama, T. Kodama, M. Makler, and J. Rafelski, Journal of Physics G: Nuclear and Particle Physics 25, 1935 (1999).

${ }^{25} \mathrm{G}$. M. Webb, J. F. McKenzie, and G. P. Zank, Journal of Plasma Physics 80, 707 (2014).

${ }^{26}$ J. D. Bekenstein and A. Oron, Physical Review E 62, 5594 (2000).

${ }^{27}$ J. D. Bekenstein and A. Oron, Foundations of Physics 31, 895 (2001).

${ }^{28}$ J. Marsden, R. Montgomery, P. Morrison, and W. Thompson, Annals of Physics 169, 29 (1986).

${ }^{29}$ E. D'Avignon, P. J. Morrison, and F. Pegoraro, Physical Review D 91, 084050 (2015).

${ }^{30}$ S. Koide, The Astrophysical Journal 696, 2220 (2009).

${ }^{31} \mathrm{We}$ remark the difference of the treatment of the velocity norm condition between preceding works of relativistic single fluid $\mathrm{AP}^{21,23,24}$. In their actions, the velocity norm condition is included in the action with Lagrange multiplier. However this method cannot be applied for generalized magnetohydrodynamic models since there are multiple velocity norm conditions for each species and the multipliers cannot be determined.

${ }^{32}$ S. Koide, The Astrophysical Journal 708, 1459 (2010).

${ }^{33}$ S. M. Mahajan, Physical Review Letters 90, 035001 (2003).

${ }^{34}$ L. Comisso and F. A. Asenjo, Physical Review Letters 113, 045001 (2014).

${ }^{35}$ F. A. Asenjo and L. Comisso, Physical Review Letters 114, 115003 (2015).

${ }^{36}$ F. A. Asenjo, L. Comisso, and S. M. Mahajan, Physics of Plasmas 22, 122109 (2015).

${ }^{37}$ H. M. Abdelhamid, Y. Kawazura, and Z. Yoshida, Journal of Physics A: Mathematical and Theoretical 48, 235502 (2015).
${ }^{38} \mathrm{M}$. Lingam, P. J. Morrison, and G. Miloshevich, Physics of Plasmas 22, 072111 (2015).

${ }^{39}$ P. J. Morrison, Rev. Mod. Phys. 70, 467 (1998).

${ }^{40}$ P. J. Morrison and J. M. Greene, Physical Review Letters 48, 569 (1982).

${ }^{41}$ P. J. Morrison, in AIP Conference Proceedings Volume 88, Vol. 88 (AIP, 1982) pp. 13-46.

${ }^{42}$ A. M. Anile, Relativistic Fluids and Magneto-Fluids (Cambridge University Press, Cambridge, 1990).

${ }^{43}$ A. Lichnerowicz, S. C. for Advanced Studies, and T. M. P. M. Series, Relativistic hydrodynamics and magnetohydrodynamics, Vol. 35 (W.A. Benjamin New York, 1967).

${ }^{44}$ F. Pegoraro, Physics of Plasmas 22, 112106 (2015).

${ }^{45} \mathrm{~F}$. Yuan, S. Markoff, and H. Falcke, Astronomy \& Astrophysics 383, 854 (2002).

${ }^{46}$ A. Taub, Physical Review 74, 328 (1948).

${ }^{47}$ G. Dixon, Special Relativity, The Foundation of Microscopic Physics (Cambridge University Press, Cambridge, 1978).

${ }^{48}$ M. Ottaviani and F. Porcelli, Physical Review Letters 71, 3802 (1993).

${ }^{49}$ L. Comisso, D. Grasso, F. L. Waelbroeck, and D. Borgogno, Physics of Plasmas 20, 092118 (2013).

${ }^{50}$ R. Lüst, Fortschritte der Physik 558, 503 (1959).

${ }^{51}$ M. Lingam, G. Miloshevich, and P. J. Morrison, Phys. Lett. A 380, 2400 (2016).

${ }^{52} \mathrm{Z}$. Yoshida, Y. Kawazura, and T. Yokoyama, Journal of Mathematical Physics 55 (2014), 10.1063/1.4872236. 